\title{
The immunoglobulin-like cell adhesion molecule hepaCAM is cleaved in the human breast carcinoma MCF7 cells
}

\author{
TING ZHANG, MEI C. MOH, LAY H. LEE and SHALI SHEN \\ Department of Physiology, Yong Loo Lin School of Medicine, National University of Singapore, 117597 Singapore
}

Received November 27, 2009; Accepted January 22, 2010

DOI: 10.3892/ijo_00000663

\begin{abstract}
We previously reported the identification and characteristics of hepaCAM, a new immunoglobulin-like adhesion molecule. Frequently lost in diverse tumors, hepaCAM exhibits antiproliferative effects on cancer cells and promotes cell-extracellular matrix (ECM) interactions when re-expressed. Herein, we demonstrate for the first time that hepaCAM is cleaved in the human breast carcinoma MCF7 cells, generating a fragment containing mainly the cytoplasmic domain. The phorbol ester phorbol 12-myristate 13-acetate (PMA) did not affect the cleavage of hepaCAM. However, calcium-influx promoted hepaCAM cleavage independent of PKC. In addition, inhibitors of proteasome and cysteine proteases strongly suppressed the cleavage of hepaCAM, indicating the involvement of proteasome, calpain-1 and cathepsin B. Furthermore, we showed that functions of hepaCAM were impaired when the cytoplasmic domain was cleaved. The truncation mutant of hepaCAM failed to promote cell-ECM adhesion and migration, and lost the inhibitory effects on cell growth, suggesting a regulatory role of the cleavage in hepaCAM functions.
\end{abstract}

\section{Introduction}

Many membrane-anchored proteins, including growth factor receptors, ligands and cell adhesion molecules (CAMs), undergo proteolytic cleavage (1). Accumulating evidence has shown that such processes have great biological and physiological significance. For example, cleavage of CD44 plays a critical role in cancer cell migration and metastasis and various physiological events (2). Proteolysis of E-cadherin regulates epithelial cell-cell adhesion, migration, and $\beta$-catenin translocation (3). Cleavage of the neural adhesion molecule

Correspondence to: Dr Shali Shen or Dr Mei C. Moh, Department of Physiology, Yong Loo Lin School of Medicine, National University of Singapore, MD9, 2 Medical Drive, 117597 Singapore

E-mail:phsssl@nus.edu.sg; phsmmc@nus.edu.sg

Key words: hepaCAM, cell adhesion molecule, proteolytic cleavage, cytoplasmic domain, cell-extracellular interactions, cell growth
L1 regulates neural cell adhesion, migration and neurite outgrowth (4).

The cleavage process of CAMs is usually differentially regulated by several pathways. It has been shown that CD44 cleavage is under the control of $\mathrm{PKC}, \mathrm{Ca}^{2+}$ influx, Rho GTPase proteins (5), membrane-type 1 matrix metalloprotease (6), and $\gamma$-secretase (7). E-cadherin cleavage is promoted by $\mathrm{Ca}^{2+}$ influx (8) and regulated by $\gamma$-secretase (9) and a disintegrin and metalloprotease 10 (ADAM10) (3). L1 is processed by ADAM10, ADAM17, and $\gamma$-secretase (4).

We have previously reported the identification of a novel gene hepaCAM in the liver (10). Located on human chromosome 11q24 and spans 7 exons, hepaCAM encodes a new member of the immunoglobulin superfamily. The predicted protein product of 416 amino acids displays a typical structure of Ig-like adhesion molecules, including an extracellular domain containing two Ig-like regions, a transmembrane segment, and a cytoplasmic tail. hepaCAM is widely expressed in normal human tissues and frequently silenced in human hepatocellular carcinoma (HCC) as well as tumors of various tissues $(10,11)$. Re-expression of hepaCAM in HepG2 (10) and MCF7 cells (12) significantly inhibits cell colony formation and retards cell growth. Furthermore, hepaCAM induces MCF7 cells to undergo cellular senescence through the p53/21 pathway (11). These results suggest a role of hepaCAM in tumor suppression. In addition, we have recently shown that hepaCAM promotes cell-ECM adhesion and cell motility through direct interaction with the actin cytoskeleton $(10,13)$.

In this study, we report for the first time that a proteolytic cleavage of hepaCAM occurs at around the transmembrane region when re-expressed in the human breast carcinoma cell line MCF7. We demonstrate that the 25-kDa hepaCAM cleavage product contains mainly the cytoplasmic domain. To identify the underlying molecular mechanisms of hepaCAM cleavage, we explored the involvement of several signaling pathways known to regulate the cleavage of CAMs. We firstly determined the effects of the phorbol ester phorbol 12-myristate 13 -acetate (PMA) and the $\mathrm{Ca}^{2+}$ ionophore ionomycin on the cleavage of hepaCAM. Using carbobenzoxyleucinyl-leucinyl-leucinal (MG132) known to inhibit mainly proteasome and also secretases and cysteine proteases, we further identified several important elements involved in hepaCAM cleavage. Finally, employing a mutant of hepaCAM with the truncation of the cytoplasmic domain, we evaluated the role of the moiety of hepaCAM after cleavage in modulating cell-ECM adhesion, cell motility and cell growth. 


\section{Materials and methods}

Antibodies and reagents. The mouse monoclonal antibodies included anti-V5 from Invitrogen (Carlsbad, CA), anticalpain-1 from Biomed Diagnostics (White city, OR), antiGAPDH, anti-HDAC2, anti-histone1 from Santa Cruz Biotechnology (Santa Cruz, CA), and anti-E-cadherin from Zymed Laboratories (San Francisco, CA). The polyclonal goat anti-lamin B and the horseradish peroxidase (HRP) conjugated goat anti-mouse secondary antibody were from Santa Cruz Biotechnology. Phorbol 12-myristate 13-acetate (PMA), EDTA, Z-Phe-Phe-Fluoromethylketone (Z-FFFMK), and CA074-Methylester (CA074-Me) were from Sigma (St. Louis, MO). GF109203X, ionomycin, Carbobenzoxyl-leucinyl-leucinyl-leucinal (MG132), ß-secretase inhibitor IV, DAPT, and MDL28170 were from Calbiochem (La Jolla, CA). Calpeptin was from Biomol International (Plymouth Meeting, PA).

Cell culture. The human breast carcinoma cell line MCF7 was obtained from American Type Culture Collection (Manassas, VA) and maintained in the recommended conditions.

Plasmid construction and transfection. The coding sequences of wild-type (WT) and mutants of hepaCAM were generated by PCR amplification. The cDNA of hepaCAM residues 1-416, residues 1-318, residues 1-263 or residues 260-416 was cloned into pcDNA6B/V5-His vector (Invitrogen) at the HindIII/ BamHI restriction sites. The constructs, namely hepaCAM, hCAM-318, hCAM-263 and hCAM-tail, were transfected into MCF7 cells using the reagent Lipofectamine Plus (Invitrogen) according to the manufacturer's instructions. For establishing stable clones, transfected cells were selected for 4 weeks in the presence of $10 \mu \mathrm{g} / \mathrm{ml}$ blasticidin (Invitrogen).

Western blot analysis. Western blot was done as previously described (10). The intensity of protein bands was quantified using GS-800 calibrated densitometer and Quantity-One software (Bio-Rad).

Cell spreading assay. Cells were seeded onto coverslips coated with $10 \mu \mathrm{g} / \mathrm{ml}$ fibronectin (Santa Cruz Biotechnology) and incubated under standard conditions. Cell morphology was examined by Zeiss Axiovert 25 inverted microscope (Germany) and photographed with a Nikon Coolpix 4500 digital camera (Japan). Un-spread cells were defined as round cells, whereas spread cells were defined as cells with extended processes (14).

Wound closure assay. Confluent monolayers of cells were wounded with a sterile plastic $200-\mu 1$ micropipette tip. The wounds were observed microscopically. The percentage of wound closure was calculated by measuring the remaining gap space on the pictures.

Colony formation. MCF7 cells transfected with pcDNA6B/ V5-His vector, hepaCAM, or hCAM-263 were selected in $10 \mu \mathrm{g} / \mathrm{ml}$ of blasticidin (Invitrogen) for 12 days without trypsinization while medium was refreshed every 2 days. The cell colonies formed at the end of experiment were stained with $1 \%$ crystal violet and observed.

Cell proliferation assay. Cells were seeded 5,000 per well in 24-well plates in triplicates and cultured for 5 days. At every $24 \mathrm{~h}$, cell viability was determined by microtetrazolium (MTT) assay. Optical density (OD) of each well was quantitated by measuring absorbance at $570 \mathrm{~nm}$. The cell number was calculated based on the following formula:

$$
\mathrm{CN}_{\mathrm{n}}=5,000 \times\left(\mathrm{OD}_{\mathrm{n}} / \mathrm{OD}_{0}\right)
$$

Where: 5,000, number of cells seeded; $\mathrm{CN}_{\mathrm{n}}$, cell number calculated on the day of observation; $\mathrm{OD}_{\mathrm{n}}, \mathrm{OD}$ measured on the day of observation; and $\mathrm{OD}_{0}$, $\mathrm{OD}$ measured on the day of seeding.

Cell cycle analysis. Cells were fixed in $70 \%$ ethanol and stored overnight at $-20^{\circ} \mathrm{C}$. Immediately before analysis, the cells were re-suspended in staining solution $(200 \mu \mathrm{g} / \mathrm{ml}$ propidium iodide, $0.1 \%$ Triton $\mathrm{X}-100$ and $2 \mathrm{mg} / \mathrm{ml} \mathrm{RNase} \mathrm{A)}$ and incubated for $30 \mathrm{~min}$ at room temperature. Cell cycle distribution was determined using a Beckman Coulter Epics Altra flow cytometer (Germany). Data were analyzed using the WinMDI (Joseph Trotter) software version 2.8.

Statistical analysis. One-way analysis of variance (ANOVA) was performed to compare the differences among more than two means. One-sample t-test was performed to compare the difference between mean of one sample and mean of the control (normalized as ' 1 '). Data were analyzed by InStat version 3.0 (GraphPad Software, Inc., La Jolla, CA). P<0.05 was considered significant.

\section{Results}

Identification of a 25-kDa species as a hepaCAM cleavage product in MCF7 cells. Gene hepaCAM encodes a type-1 transmembrane Ig-like adhesion molecule of 416 amino acids, consisting of an extracellular domain, a trans-membrane domain, and a cytoplasmic domain (Fig. 1A, hepaCAM). Cloned into the eukaryotic expression vector pcDNA6B/V5His, hepaCAM was re-expressed in the human breast carcinoma MCF7 cells, a hepaCAM deficient cell line. V5 epitope was tagged at the cytoplasmic $\mathrm{COOH}$-terminus of hepaCAM, which allowed detection of hepaCAM protein with anti-V5 antibody. The molecular mass of the V5-tagged hepaCAM was approximately $75 \mathrm{kDa}$.

In addition to the full-length protein of hepaCAM, a band of approximately $25 \mathrm{kDa}$ was identified. The $25-\mathrm{kDa}$ band represented a fragment including the cytoplasmic $\mathrm{COOH}-$ terminus as it was revealed by anti-V5 antibody (Fig. 1B, lane 2). Truncated between the residues 319 and 416 (98 amino acids deleted), the mutant hCAM-318 showed a molecular mass at about $60 \mathrm{kDa}$ and an additional band at about $10 \mathrm{kDa}$ (Fig. 1B, lane 3), proving that the 25-kDa species was indeed derived from hepaCAM.

Furthermore, to determine if the $25-\mathrm{kDa}$ fragment contained the cytoplasmic domain, we constructed another mutant of hepaCAM with the cytoplasmic domain only (residues 260-416), designated as hCAM-tail (Fig. 1A, hCAMtail). The cytoplasmic domain alone shared a similar molecular 
A

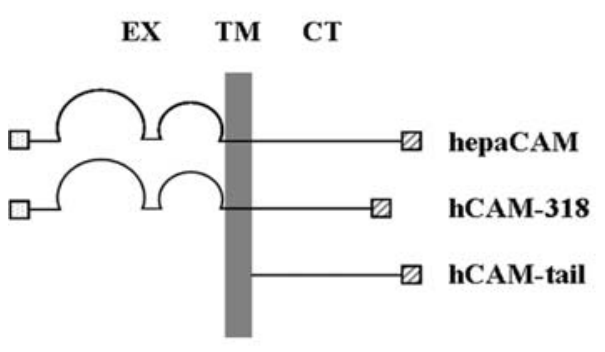

B

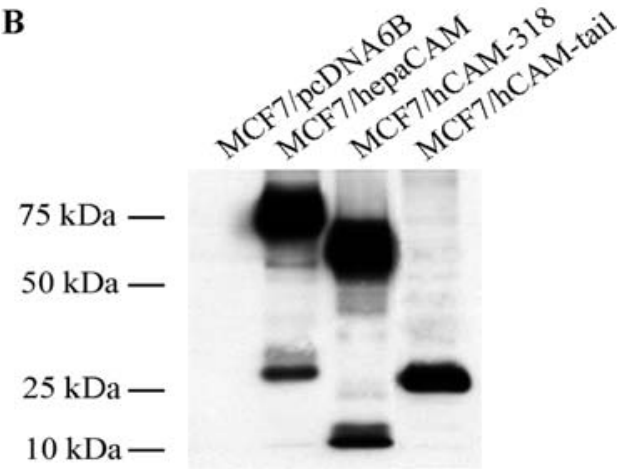

Figure 1. hepaCAM undergoes a proteolytic cleavage generating a product containing mainly the cytoplasmic domain. (A) Sequences of the WT and truncation mutants of hepaCAM were cloned into the eukaryotic expression vector pcDNA6B/V5-His and transfected into the breast carcinoma cell line MCF7. EX, extracellular domain; TM, transmembrane domain; CT, cytoplasmic domain; stripes, signal peptitde; dots, V5 tag. (B) Protein expression of pcDNA6B/V5-His and V5-fused WT and mutant hepaCAM (hCAM-318 and hCAM-tail) in MCF7 cells was examined by Western blot analysis using anti-V5 antibody.

mass with the cleaved fragment (Fig. 1B, lane 4), suggesting that the $25-\mathrm{kDa}$ cleavage product contained mainly the cytoplasmic domain of hepaCAM.

PMA, a phorbol ester, has no effect on hepaCAM cleavage. Phorbol esters have been reported to facilitate proteolytic cleavage of numerous cell surface proteins, such as E-cadherin (3) and CD44 (5). To determine if phorbol esters could promote hepaCAM cleavage, the effects of PMA, one of the most commonly used phorbol esters, was examined. MCF7 cells stably expressing hepaCAM were treated with PMA for 15, 30, 60 and $120 \mathrm{~min}$. As shown in Fig. 2, PMA up-regulated both the full-length and the cleavage product of hepaCAM. Quantification of the intensities of the protein bands revealed that the ratio of the cleavage product versus the full-length of hepaCAM remained constant through out the time courses, indicating that the degree of hepaCAM cleavage was not altered by PMA.

$\mathrm{Ca}^{2+}$ influx promotes hepaCAM cleavage. Calcium activities have been shown to be involved in the processing of many trans-membrane proteins $(5,8)$. To examine if $\mathrm{Ca}^{2+}$ influx could induce hepaCAM cleavage, MCF7/hepaCAM cells were treated with the $\mathrm{Ca}^{2+}$ ionophore ionomycin. The results showed that ionomycin caused a prominent accumulation of the cleavage product of hepaCAM, while the full-length of hepaCAM was correspondingly reduced (Fig. 3A). However, when the cells were co-treated with the $\mathrm{Ca}^{2+}$ chelator EDTA,
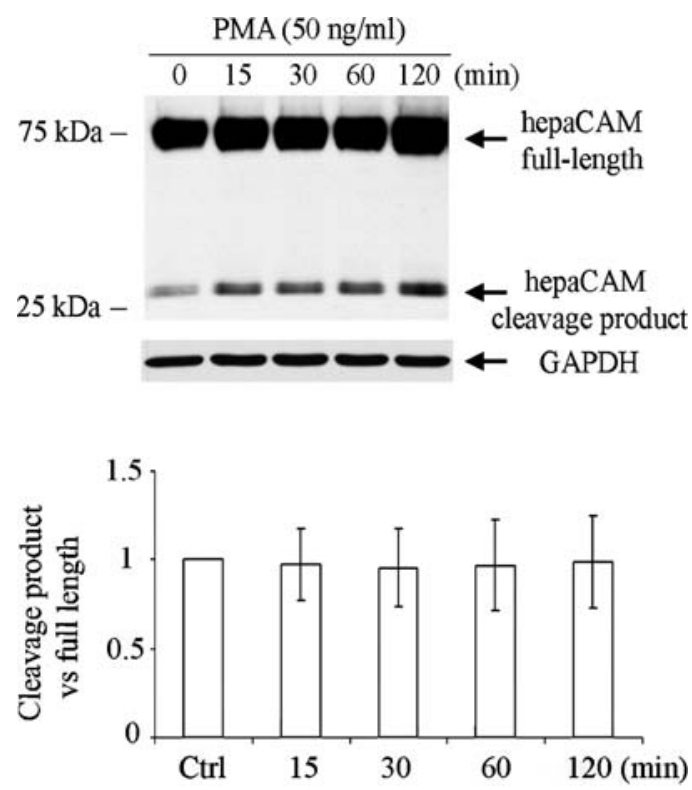

Figure 2. PMA does not affect hepaCAM cleavage. MCF7/hepaCAM cells were incubated with PMA $(50 \mathrm{ng} / \mathrm{ml})$ for $0,15,30,60$ or $120 \mathrm{~min}$. Cell lysates were analyzed by Western blot analysis with anti-V 5 antibody. GAPDH served as the loading control. Intensities of protein bands were measured, and the ratios of cleavage product against full-length of hepaCAM (mean $\pm \mathrm{SD}, \mathrm{n}=3$ ) are represented in the bar chart. Positions of the molecular size markers are shown on the left of the panel.

the ionomycin-induced hepaCAM cleavage was diminished (Fig. 3B, lane 4-6). These data indicated that the effect of ionomycin on hepaCAM cleavage was dependent on the influx of $\mathrm{Ca}^{2+}$ across the plasma membrane followed by the elevation of the intracellular $\mathrm{Ca}^{2+}$ level. In addition, the treatment of EDTA alone had no effects on the cleavage of hepaCAM (Fig. 3B, lane 3), suggesting that the extracellular $\mathrm{Ca}^{2+}$ was not directly involved in the cleavage of hepaCAM.

One of the consequences of $\mathrm{Ca}^{2+}$ influx is the activation of protein kinase $\mathrm{C}$ (PKC) $(15,16)$. To examine the role of $\mathrm{PKC}$ in ionomycin-induced hepaCAM cleavage, MCF7/ hepaCAM cells were pretreated with the PKC inhibitor GF109203X followed by the treatment with ionomycin. As shown in Fig. 3C, GF109203X had no effect on ionomycinstimulated cleavage of hepaCAM, implying that the $\mathrm{Ca}^{2+}$ influx-induced cleavage was independent of PKC pathway.

Proteasome inhibitors suppress hepaCAM cleavage. It has been shown that cleavage products of a number of proteins are immediately degraded through the ubiquitin-proteasome system. For example, the cleavage product of CD44 is undetectable without the presence of proteasome inhibitor (5). To investigate if the $25-\mathrm{kDa}$ cleavage product of hepaCAM undergoes proteasomal degradation, MCF7/hepaCAM cells were treated with a proteasome inhibitor MG132. Intriguingly, instead of being accumulated, the cleavage product of hepaCAM was significantly reduced after MG132 treatment in a time dependent manner (Fig. 4A). This unexpected result indicated that the cleavage process of hepaCAM was rather distinct from that of other CAMs.

It is known that MG132 is not a specific inhibitor of proteasome. In addition to proteasome, MG132 has a broad spectrum 
A
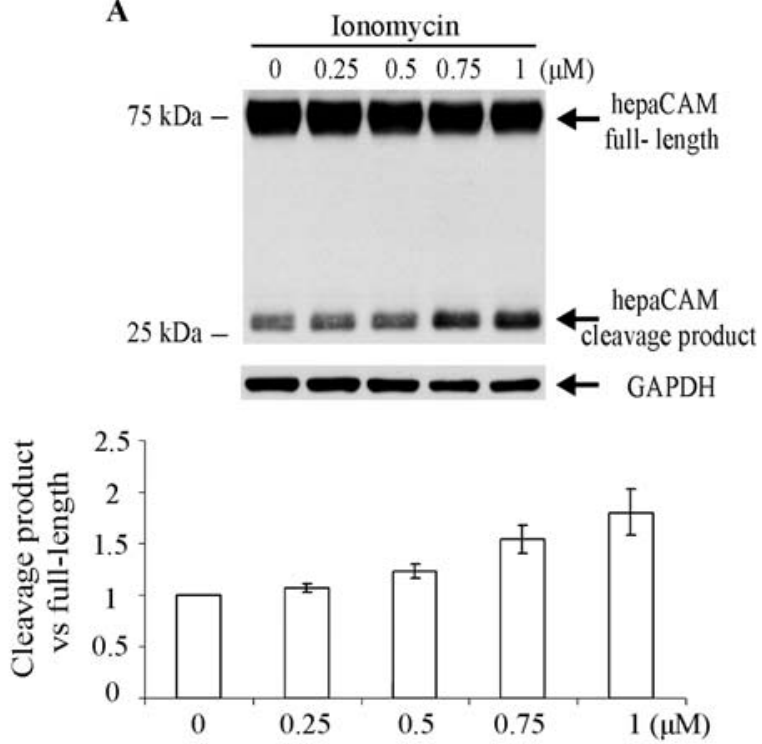

B

C
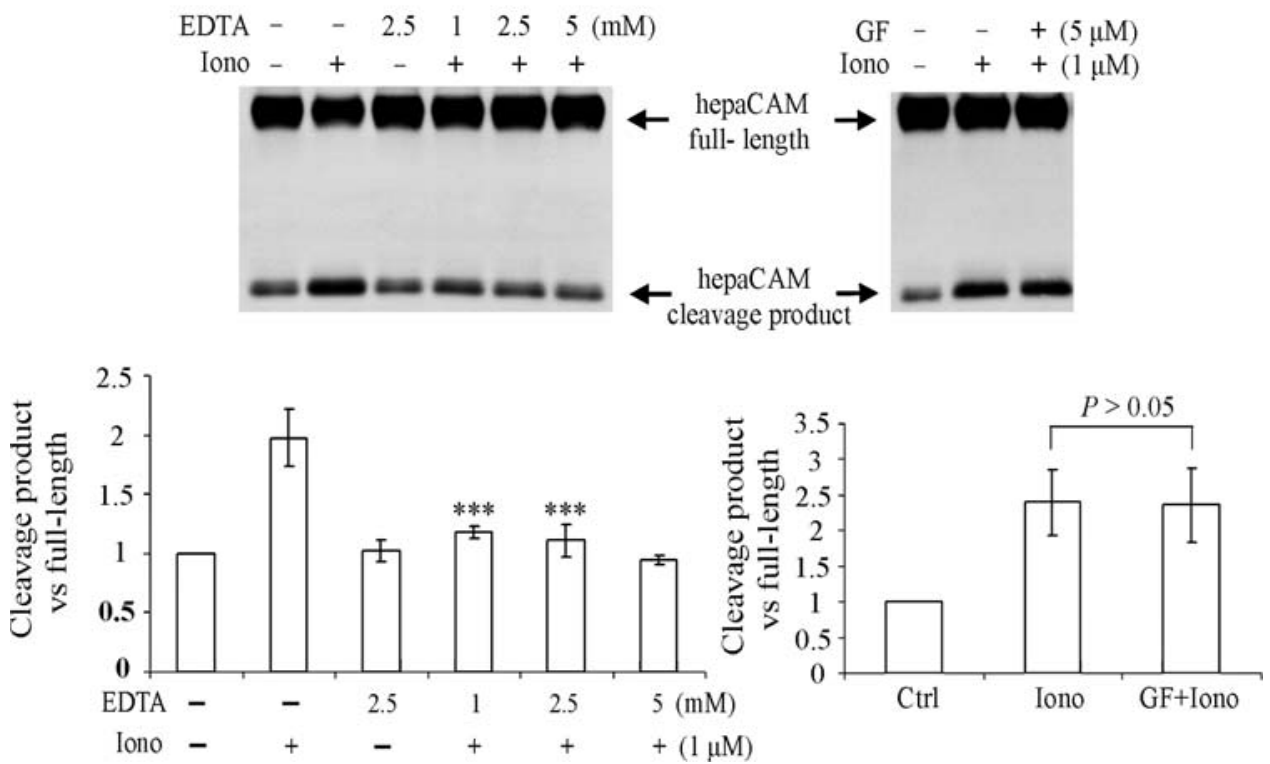

Figure 3. Calcium influx promotes hepaCAM cleavage. MCF7/hepaCAM cells were incubated with ionomycin at indicated concentrations (A) or coincubated with ionomycin $(1 \mu \mathrm{M})$ and/or EDTA at indicated concentrations (B) for $20 \mathrm{~min}$ at $\mathrm{pH} 8.5$ or pre-incubated with (lane 3) or without (lane 2) GF109203X $(5 \mu \mathrm{M})$ for $1.5 \mathrm{~h}$ before addition of ionomycin $(1 \mu \mathrm{M})$ for $20 \mathrm{~min}(\mathrm{C})$. Iono, ionomycin; GF, GF109203X. Cell lysates were analyzed by Western blot analysis with anti-V5 antibody. GAPDH served as the loading control. The ratios of cleavage product against full-length of hepaCAM (mean \pm SD, $n=3$ ) are represented in the bar chart. ${ }^{* *} \mathrm{P}<0.01 ;{ }^{* * *} \mathrm{P}<0.001$. Positions of the molecular size markers are shown on the left of each panel.

of inhibitory effects on proteases, including $\beta$ - and $\gamma$-secretase $(17,18)$ and cysteine proteases $(17)$. To evaluate the involvement of proteasome, MCF7/hepaCAM cells were treated with the specific proteasome inhibitor clasto-lactacystin B-lactone (mentioned as lactacystin in the subsequent text). The result showed that not only MG132 but also lactacystin significantly decreased the cleavage product with an increase in the fulllength of hepaCAM (Fig. 4B, lanes 2 and 3), suggesting that proteasome was involved in the cleavage of hepaCAM.

Next, to evaluate the possible participation of other enzymes that were inhibited by MG132, MCF7/hepaCAM cells were treated with $\beta$-secretase inhibitor IV and the $\gamma$ secretase inhibitor DAPT. Interestingly, none of these agents reduced the extent of hepaCAM cleavage. Conversely, Bsecretase inhibitor IV resulted in an apparent increase of
hepaCAM cleavage while $\gamma$-secretase inhibitor DAPT had no significant effect on hepaCAM (Fig. 4B, lanes 4 and 5). These results indicated that $\beta$ - and $\gamma$-secretase were not responsible for the MG132-induced suppression of hepaCAM cleavage.

Members of cysteine proteases, calpain-1 and cathepsin B, are involved in hepaCAM cleavage. As MG132 also inhibits cysteine proteases calpain and cathepsin, we investigated the involvement of these enzymes in hepaCAM cleavage. Two calpain inhibitors MDL28170 and calpeptin significantly reduced the cleavage product and accumulated the full-length of hepaCAM (Fig. 5A). As MCF7 cells are deficient of calpain-2 (19), we assayed the activation status of calpain-1. In the inactive condition, calpain-1 exists as pro-enzyme heterodimer of 80-29 kDa, and following $\mathrm{Ca}^{2+}$ binding undergoes 

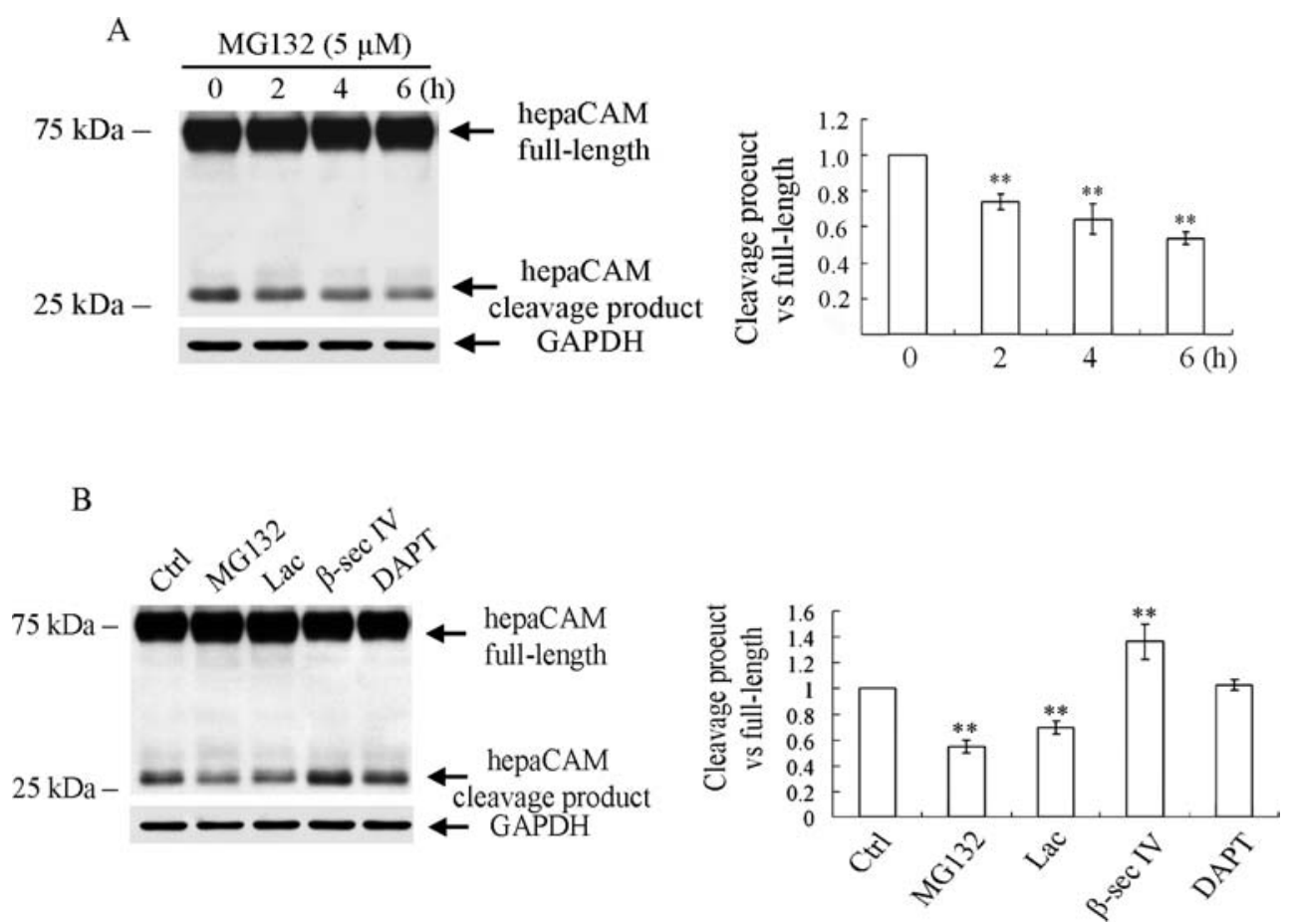

Figure 4. hepaCAM cleavage is suppressed by inhibitors of proteasome. MCF7/hepaCAM cells were incubated with MG132 $(5 \mu \mathrm{M})$ for indicated time courses (A) or MG132 $(5 \mu \mathrm{M})$, B-secretase inhibitor IV $(10 \mu \mathrm{M})$, DAPT $(10 \mu \mathrm{M})$, pepstatin A methyl ester $(20 \mu \mathrm{m})(\mathrm{B})$ for 16 h. Cell lysates were analyzed by Western blot analysis with anti-V5 antibody. GAPDH served as the loading control. B-sec IV, B-secretase inhibitor IV; PAME, pepstatin A methyl ester. The ratios of cleavage product against full-length of hepaCAM (means $\pm \mathrm{SD}, \mathrm{n}=3$ ) are represented in the bar chart. ${ }^{* *} \mathrm{P}<0.01$. Positions of the molecular size markers are shown on the left of each panel.

autolysis to an active 78-18 $\mathrm{kDa}$ heterodimer (20). Western blot revealed the presence of both 78 - and $80-\mathrm{kDa}$ species of calpain-1 large subunit in untreated cells (Fig. 5B, lane 1), indicating a basal activity of this enzyme. Following treatment of MDL28170 and calpeptin, the autolysis of calpain-1 was prevented, and a clear accumulation of the $80-\mathrm{kDa}$ large subunit was observed (Fig. 5B, lanes 2 and 3). These results provided strong evidence that calpain-1 may be involved in hepaCAM cleavage.

The cysteine proteases cathepsin B and L have been reported to be inhibited by MG132 $(21,22)$. We showed that two cathepsin inhibitors Z-FF-FMK (cathepsin B and L inhibitor) and CA074-Me (cathepsin B inhibitor) efficiently decreased hepaCAM cleavage (Fig. 5C). Taken together, these data suggested that calpain-1 and cathepsin B, members of cysteine proteases, were involved in the cleavage of hepaCAM.

The cytoplasmic domain is essential for hepaCAM-mediated cell-ECM adhesion and cell motility. We have demonstrated that the cleavage product of hepaCAM contains mainly the cytoplasmic domain. To evaluate the functional role of the moiety of hepaCAM after cleavage, a mutant with the truncation of the entire cytoplasmic domain (residues 264-416) was constructed. Designated as hCAM-263, this mutant was stably expressed in MCF7 cells (Fig. 6A). The adhesive property of MCF7 cells expressing pcDNA6B vector, WT hepaCAM or hCAM-263 on fibronectin was evaluated through cell adhesion assay. Compared to that of the control MCF7/ pcDNA6B cells 15 and 52\%, the spreading of MCF7/
hepaCAM cells was 35 and 74\% $(\mathrm{P}<0.001)$ and MCF7/ hCAM-263 cells 11 and $47 \%$ ( $P>0.05)$, at $30 \mathrm{~min}$ and $2 \mathrm{~h}$ of incubation, respectively (Fig. 6B). These results suggested that when the cytoplasmic domain was truncated, hepaCAM lost the ability to promote cell-ECM adhesion.

Motility of MCF7 cells expressing pcDNA6B vector, WT hepaCAM or hCAM-263 was assessed by wound closure assay. Confluent monolayers of cells were wounded and allowed to heal. After $24 \mathrm{~h}$ of incubation, the wound closure was $56 \%$ by MCF7/hepaCAM whereas $32 \%$ by MCF7/ hCAM-263 cells and $25 \%$ by the control MCF7/pcDNA6B cells. After $48 \mathrm{~h}$, the wound closure reached $86 \%$ by MCF7/ hepaCAM cells whereas $59 \%$ by MCF7/hCAM-263 cells and $51 \%$ by the control MCF7/pcDNA6B cells (Fig. 7A). MCF7/ hepaCAM cells displayed the fastest wound closure $(\mathrm{P}<0.001)$ and the closure rate of MCF7/hCAM-263 cells was comparable ( $\mathrm{P}>0.05)$ to that of MCF7/pcDNA6B cells. Moreover, at $3 \mathrm{~h}$, $\mathrm{MCF} 7 /$ hepaCAM cells at the edge of the wound showed noticeable cell protrusions (Fig. 7B, middle panel, arrowheads), which marked the start of cell migration. When the cytoplasmic domain was deleted, such protrusions were not observed (Fig. 7B, right panel), similar to the control cells (Fig. 7B, left panel). These results indicated that when the cytoplasmic domain was cleaved, hepaCAM was not able to enhance cell motility.

The cytoplasmic domain plays a key role in the growth inhibitory effect of hepaCAM. To study the role of the cytoplasmic domain in cell growth controlled by hepaCAM, MCF7 cells were transfected with either WT hepaCAM or 

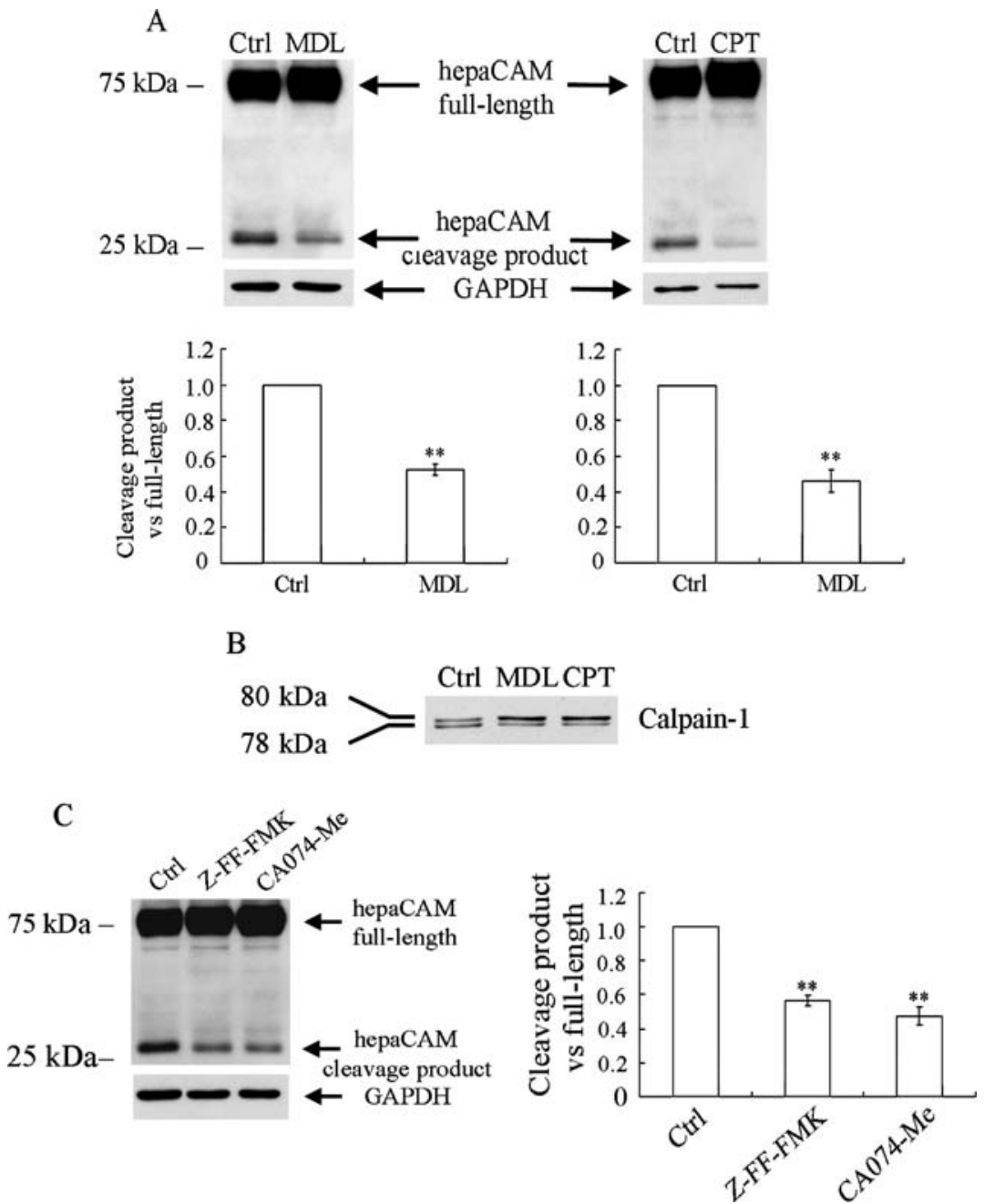

Figure 5. hepaCAM cleavage is inhibited by calpain and cathepsin inhibitors. MCF7/hepaCAM cells were incubated with MDL28170 (100 $\mu \mathrm{M})$, calpeptin $(50 \mu \mathrm{M})(\mathrm{A})$ or Z-FF-FMK $(40 \mu \mathrm{M})$ and CA074-Me $(40 \mu \mathrm{M})(\mathrm{C})$ for $16 \mathrm{~h}$. Cleavage of hepaCAM and calpain-1 activation status were analyzed by Western blot analysis with anti-V5 antibody and (B) anti-calpain-1 antibody, respectively. GAPDH served as the loading control. MDL, MDL28170; CPT, calpeptin. The ratios of cleavage product against full-length of hepaCAM (means $\pm \mathrm{SD}, \mathrm{n}=3$ ) are represented in the bar chart. ${ }^{* *} \mathrm{P}<0.01$. Positions of the molecular size markers are shown on the left of each panel.

hCAM-263. Cells transfected with pcDNA6B vector served as the control. After 12 days of antibiotic selection, the colonies formed were stained with crystal violet for observation (Fig. 8A). Compared to the vector control, expression of hepaCAM strongly reduced both the number and the size of cell colonies. The expression of hCAM-263 less significantly inhibited colony formation. Furthermore, the growth rate of MCF7/pcDNA6B, MCF7/hepaCAM or MCF7/hCAM-263 cells was determined by MTT cell proliferation assay (Fig. 8B). Compared to that of MCF7/pcDNA6B cells, the growth rate of $\mathrm{MCF} 7 /$ hepaCAM was significantly slower $(\mathrm{P}<0.01)$ on day 5. However, hCAM-263 did not clearly inhibit the growth of MCF7 cells. These results implied that the inhibitory effect of hepaCAM on cell growth was diminished when the cytoplasmic domain was cleaved.

To study if the antiproliferative effect of hepaCAM was a consequence of cell cycle arrest, flow cytometry was carried out to determine the cell cycle distribution of the cells. Intriguingly, MCF7/hepaCAM cells displayed a unique cell cycle profile that $83 \%$ of the cell population was accumulated in $\mathrm{G} 2 / \mathrm{M}$ phase (Fig. $8 \mathrm{C}$, histogram, middle panel), indicating a significant G2/M cell cycle arrest. However, MCF7 cells expressing hCAM-263 displayed a cell cycle profile similar to that of the control cells, suggesting that the cytoplasmic domain was critical for the regulation of cell cycle by hepaCAM.

\section{Discussion}

In our previous studies, we identified a novel Ig-like cell adhesion molecule designated hepaCAM and revealed its tumor-suppressing properties. In the current study, we have demonstrated for the first time that hepaCAM undergoes a proteolytic cleavage when exogenously expressed in MCF7 cells. It would be valuable to study the cleavage pattern of endogenous hepaCAM in other cancer cell lines. However, as we have reported previously, this gene is frequently lost in diverse human tumors (10). Moreover, the cytoplasmic domain of hepaCAM is highly phosphorylated (12) making it difficult to generate antibodies against the cytoplasmic region. Therefore, our research was based on transfection studies on the hepaCAM deficient cell line MCF7 and detection of the V5 tagged hepaCAM by anti-V5 antibody. 
A
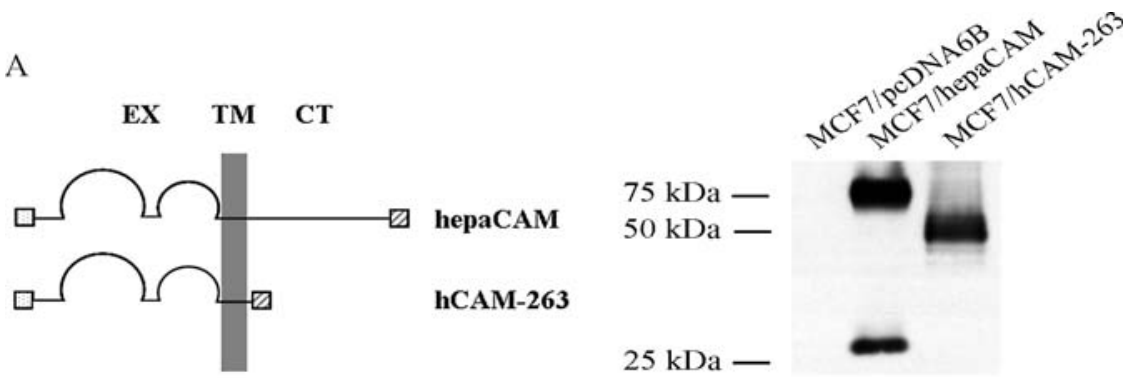

B
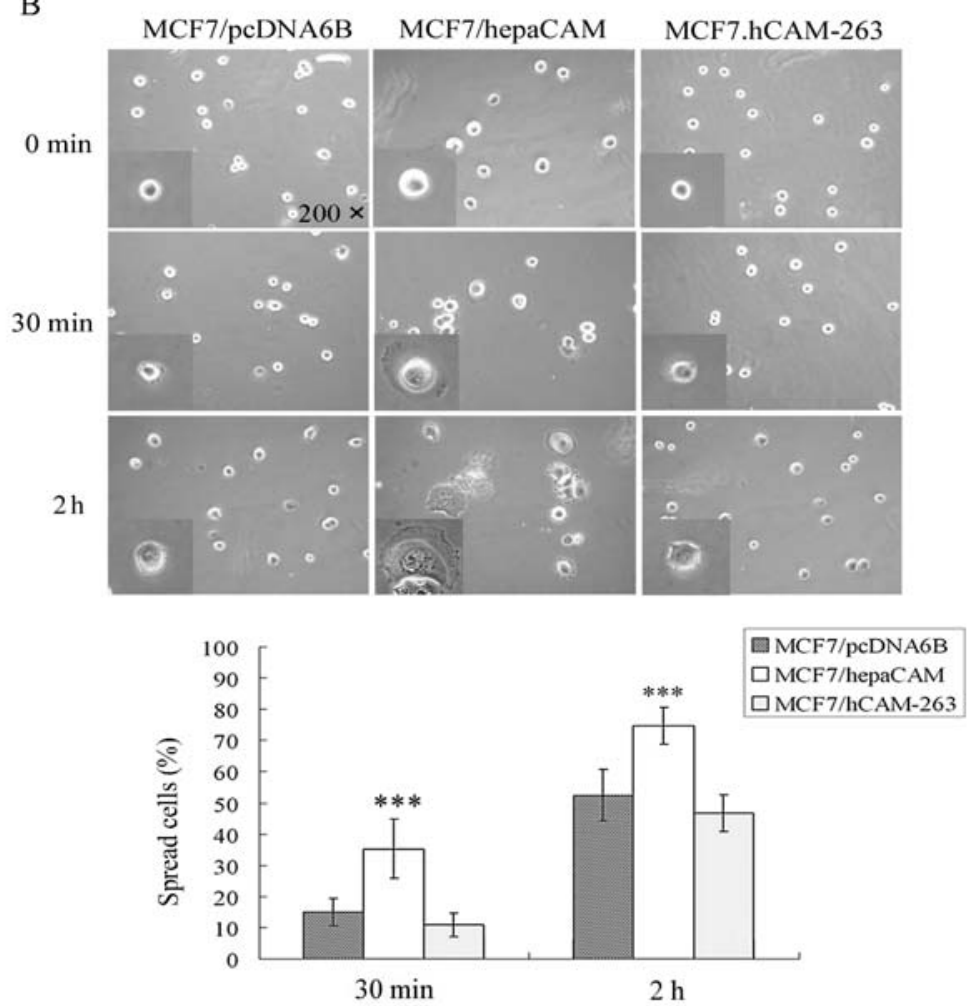

Figure 6. Effects of cytoplasmic domain truncation on hepaCAM-mediated cell-ECM adhesion. (A) Sequence of the hepaCAM mutant hCAM-263 was cloned into the eukaryotic expression vector pcDNA6B/V5-His and stably transfected into MCF7 cells. Protein expression of pcDNA6B/V5-His vector, V5-fused WT hepaCAM and hCAM-263 in MCF7 cells was examined by Western blot using anti-V5 antibody. Positions of the molecular size markers are shown on the left of the panel. (B) Adhesion of MCF7/pcDNA6B, MCF7/hepaCAM and MCF7/hCAM-263 cells on fibronectin was determined by cell adhesion assay. The microscopic photos were taken under x200 magnification at $30 \mathrm{~min}$ and $2 \mathrm{~h}$ after seeding. Insets, cell morphology. Percentages of spread cells determined in 5 randomly selected fields (means $\pm \mathrm{SD}, \mathrm{n}=5$ ) are represented in the bar chart. ${ }^{* * *} \mathrm{P}<0.001$.

We have found that the magnitude of hepaCAM cleavage is not altered by PMA, a phorbol ester that has been widely documented to stimulate cleavage of cell surface proteins $(23,24)$. Exposures to PMA up to $2 \mathrm{~h}$ only up-regulated the total protein expression of hepaCAM. Prolonged treatment with PMA up to $24 \mathrm{~h}$ (data not shown) further enhanced the expression level and resulted in an increase of the ratio between the cleavage product over the full-length of hepaCAM. However, this increase was probably due to the saturation of the full-length protein at later time points. It has been reported that the effect of PMA on proteolytic processing is rapid (25); therefore we concluded that PMA has no effect on hepaCAM cleavage.

In addition, we showed that $\mathrm{Ca}^{2+}$ influx induced by ionomycin promotes hepaCAM cleavage. Such effect can be diminished by the $\mathrm{Ca}^{2+}$ chelator EDTA, supporting the role of
$\mathrm{Ca}^{2+}$ influx in inducing hepaCAM cleavage. Elevation in intracellular $\mathrm{Ca}^{2+}$ concentration is known to activate PKC $(15,16)$. However, the PKC inhibitor GF109203X failed to exhibit any inhibitory effects on ionomycin-induced hepaCAM cleavage, implying that the downstream PKC activation is not required for activation of this $\mathrm{Ca}^{2+}$ influx-initiated pathway.

Unlike other CAMs, hepaCAM cleavage product is significantly reduced when proteasome is inhibited, suggesting that proteasome is involved in the cleavage of hepaCAM. It is of note that the main function of proteasome is to degrade unneeded proteins by non-specific proteolysis (26). On the other hand, recent studies have shown that proteasome can cleave its substrate in a specific manner. Sorokin et al (27) have demonstrated that Y-box-binding protein 1 (YB-1) undergoes a limited proteolysis by the $20 \mathrm{~S}$ proteasome. Other examples of proteins undergoing limited proteasomal 
A
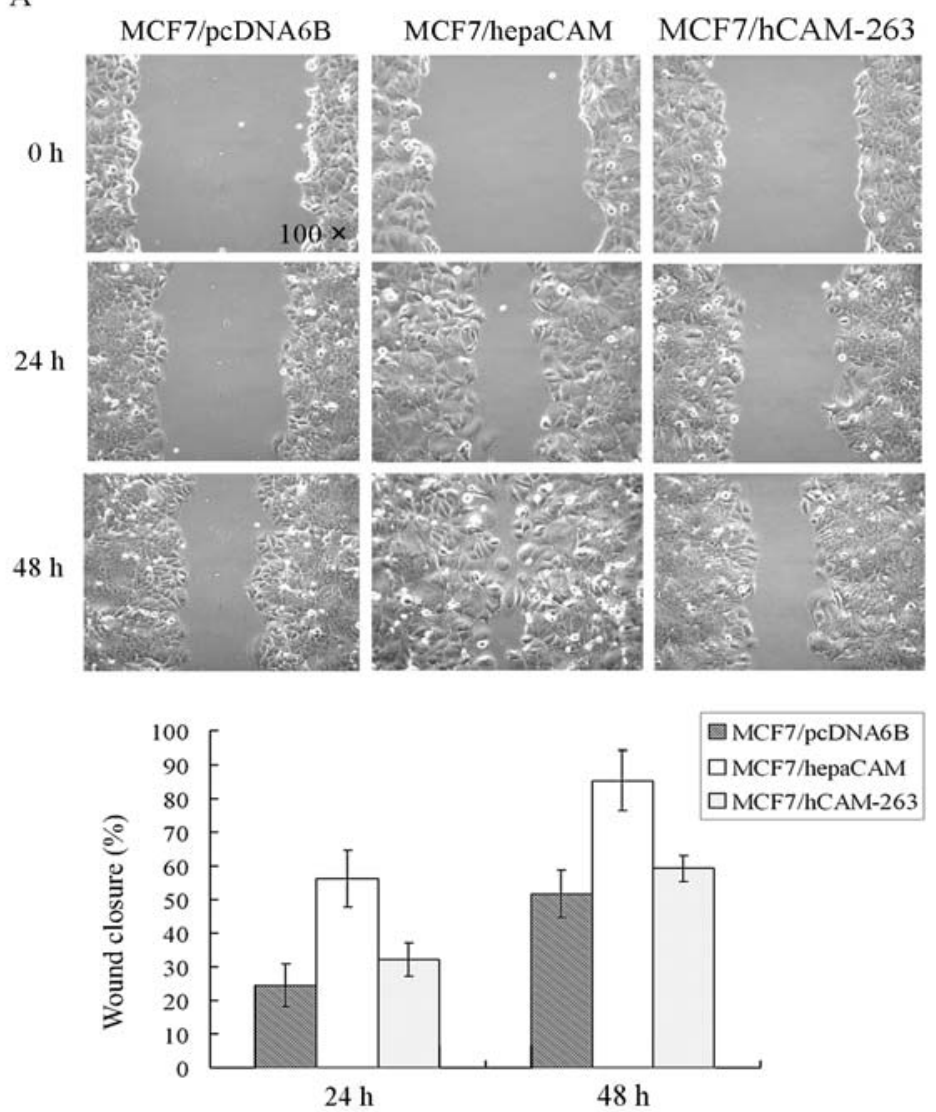

B
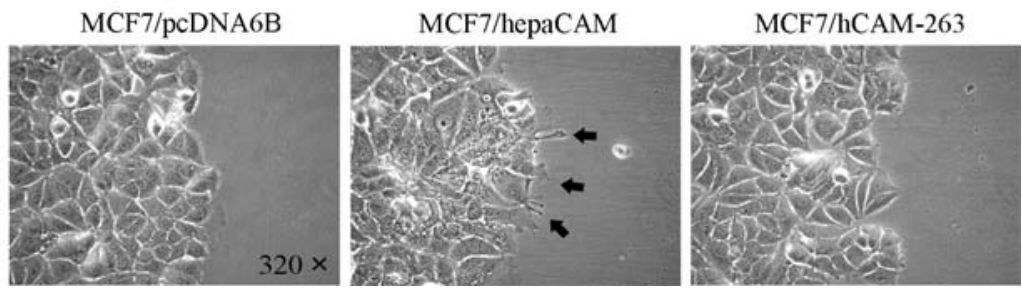

Figure 7. Effects of cytoplasmic domain truncation on hepaCAM-mediated cell motility. (A) Confluent monolayers of MCF7/pcDNA6B, MCF7/hepaCAM and MCF7/hCAM-263 cells were wounded and allowed to heal. The microscopic photos were taken under x100 magnification at 24,48 and $72 \mathrm{~h}$ after wounding. Wound closure computed in percentages (means $\pm \mathrm{SD}, \mathrm{n}=6$ ) are represented in the bar chart. ${ }^{* * *} \mathrm{P}<0.001$. (B) Microscopic photos were taken at the edges of wounds under x320 magnification $3 \mathrm{~h}$ after wounding. Protrusions of MCF7/hepaCAM cells are indicated by arrowheads.

cleavage include the translation initiation factors eIF4G and eIF3a (28). The mechanism of hepaCAM cleavage mediated by proteasome remains unknown.

Besides proteasome, MG132 exhibits inhibitory effects on other enzymes. It has been reported that MG132 inhibits the cleavage of Notch-1 by $\gamma$-secretase (29). Brown et al have shown that $\gamma$-secretase is involved in the regulated intramembranous proteolysis (RIP) (30). RIP cleaves only type-1 transmembrane protein (31) and does not depend on sequence recognition (32). We have shown that hepaCAM is type-1 transmembrane protein (10), offering a clue that hepaCAM may be a substrate of RIP. However, $\gamma$-secretase inhibitor failed to suppress hepaCAM cleavage, ruling out that hepaCAM is a RIP substrate. It has also been shown that MG132 blocks maturation of the amyloid precursor protein Swedish mutant by preventing its cleavage by $\beta$-secretase (17). Interestingly, the inhibitor of $\beta$-secretase leads to an accumulation of the cleavage product, suggesting that, as a negative regulator, $\beta$-secretase may indirectly be involved in hepaCAM cleavage.

Cysteine proteases are a family of enzymes that have a common mechanism involving a nucleophilic cysteine thiol in a catalytic triad. Among members of cysteine protease family, calpain as well as cathepsin B and L can be inhibited by MG132 (33). Using inhibitors of calpain and cathepsin, we showed the possible involvement of calpain-1 and cathepsin B in hepaCAM cleavage.

Collectively, we have demonstrated several pathways that regulate hepaCAM cleavage. The existence of multiple pathways for the regulation of proteolytic cleavage have been shown in other membrane proteins, including transforming growth factor- $\alpha$ (35), kit ligand (38), c-kit receptor (36), and heparin-binding epidermal growth factor (37). Each pathway may be responsible for a particular intracellular or extracellular 
A

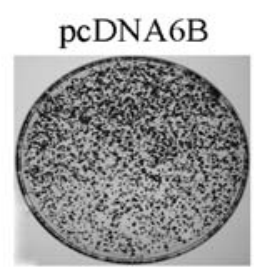

hepaCAM
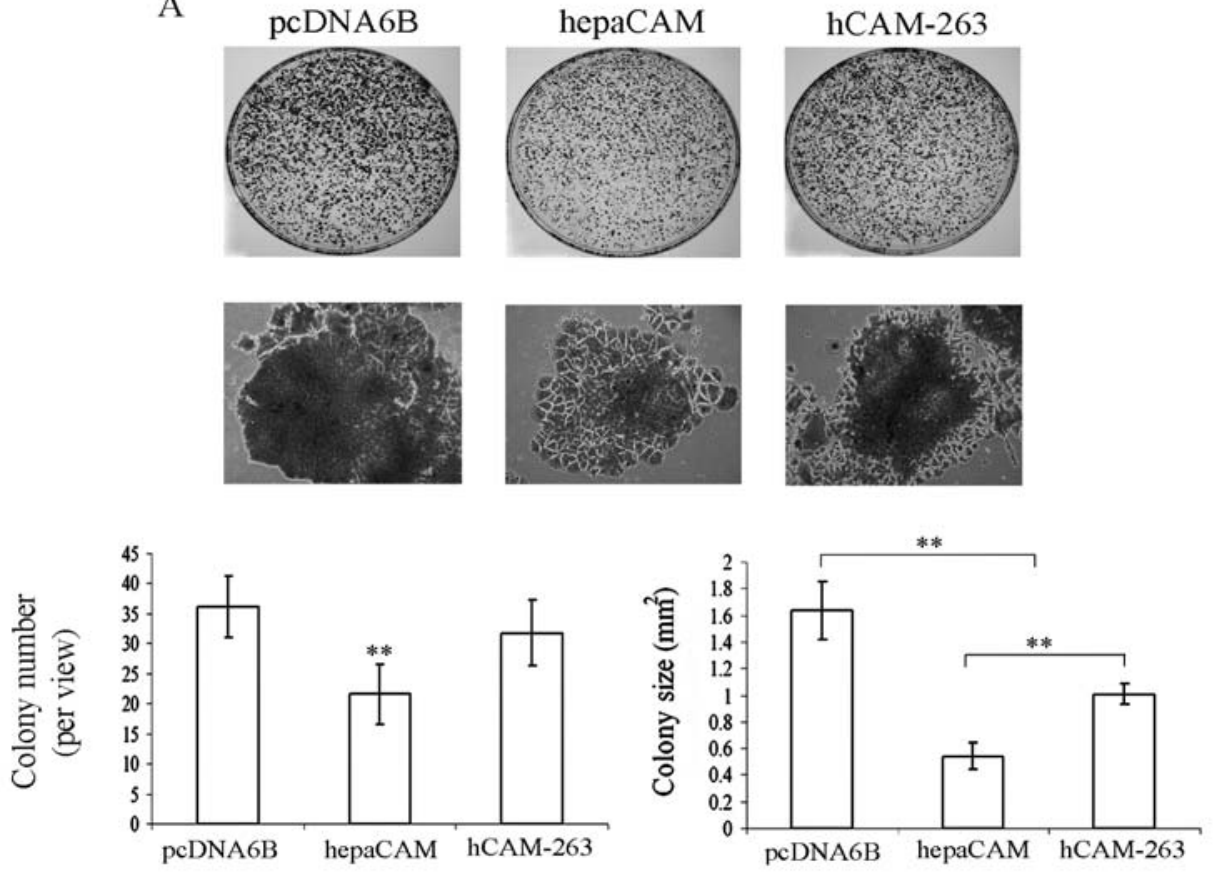

$\mathrm{B}$
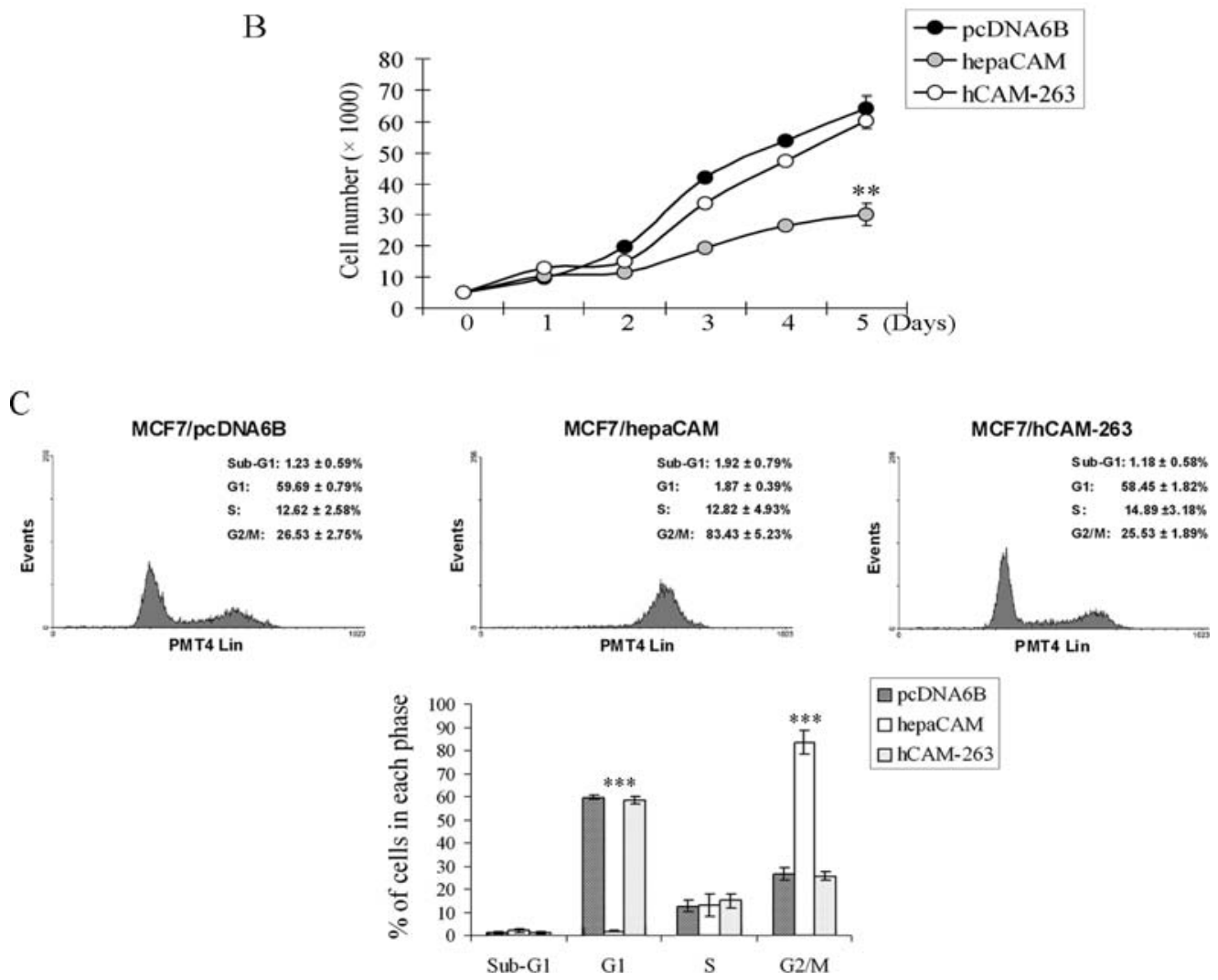

$\square$ hepaCAM

口 hCAM-263

Figure 8. Effects of cytoplasmic domain truncation on hepaCAM-mediated cell growth inhibition. (A) MCF7 cells were transfected with pcDNA6B/V5-His vector, hepaCAM or hCAM-263 and selected in $10 \mu \mathrm{g} / \mathrm{ml}$ blasticidin for 12 days. The cell colonies formed at the end of the experiment were stained with $1 \%$ crystal violet (top row). Microscopic photos of the average cell density in a stained colony were taken at x100 magnification (middle row). The number and size of colonies (means $\pm \mathrm{SD}, \mathrm{n}=5$ ) are represented in the bar charts. ${ }^{* *} \mathrm{P}<0.01$. (B) Growth rate of MCF7/pcDNA6B, MCF7/hepaCAM and MCF7/hCAM-263 cells was monitored by MTT assay for 5 days. Data represent means \pm SD of three experiments performed in triplicates. ${ }^{* *} \mathrm{P}<0.01$. (C) Cell cycle distribution of MCF7/pcDNA6B, MCF7/hepaCAM and MCF7/hCAM-263 cells was determined by flow cytometry. Percentages of cells in each phase (means \pm SD, $n=3$ ) are represented in the bar chart. ${ }^{* * *} \mathrm{P}<0.001$.

signal, so that hepaCAM could respond to a variety of stimulations in a complex physiological or pathological environment.

An interesting feature of hepaCAM cleavage is that it gives rise to a cleavage product containing mainly the cyto- plasmic domain. We have previously demonstrated that hepaCAM exhibits anti-proliferative effects on cancer cell lines and promotes cell-extracellular matrix (ECM) interactions $(10,12)$. Here we have further shown that truncation 
of the cytoplasmic domain largely reduced the effects of hepaCAM on cell-ECM adhesion and cell motility. Moreover, the mutant hepaCAM without the cytoplasmic domain has lost the inhibitory effects on cell growth and failed to induce G2/M cell cycle arrest. The importance of the cytoplasmic domain has been emphasized in the functional regulation of transmembrane proteins. Alterations of several CAMs at the cytoplasmic region have led to dysfunction of the proteins. One such CAM is the tumor suppressor in lung cancer 1 (TSLC1), which harbors the 4.1 ezrin/radixin/moesin (FERM) and PSD-95/Dig/ZO-1 (PDZ)-interacting motif in the cytoplasmic domain. Deletion of these motifs abrogates tumor suppressor activity of TSLC1 (38). Another example is CD44. Using the truncation mutants lacking part of the cytoplasmic domain, Jiang et al have shown that the CD44 cytoplasmic domain is required for hyaluronan binding, pericellular matrix assembly, and receptor-mediated endocytosis in COS-7 cells (39). Our findings on hepaCAM reiterate the importance of the cytoplasmic domain in the functions of transmembrane proteins.

In conclusion, our results indicate that hepaCAM cleavage is a complex process. Various molecular elements representing several signaling pathways are involved in hepaCAM cleavage. The proteolytic cleavage may serve as a mechanism to regulate hepaCAM functions in response to different intracellular or extracellular signals.

\section{Acknowledgements}

We thank Ms. Asha Reka Das for her assistance wherever and whenever needed. This study was supported by the National University of Singapore Academic Research Fund Grant R-185-000-169-112 and the Singapore Millennium Foundation Postdoctoral Fellowship Grant (M.C. Moh).

\section{References}

1. Gordon MY: Hemopoietic growth factors and receptors: bound and free. Cancer Cell 3: 127-133, 1991.

2. Okamoto I, Kawano Y, Murakami D, et al: Proteolytic release of CD44 intracellular domain and its role in the CD44 signaling pathway. J Cell Biol 155: 755-762, 2001.

3. Maretzky T, Reiss K, Ludwig A, et al: ADAM10 mediates Ecadherin shedding and regulates epithelial cell-cell adhesion, migration, and B-catenin translocation. Proc Natl Acad Sci USA 102: 9182-9187, 2005.

4. Maretzky T, Reiss K, Ludwig A, et al: L1 is sequentially processed by two differently activated metalloproteases and presenilin/ gamma-secretase and regulates neural cell adhesion, cell migration, and neurite outgrowth. Mol Cell Biol 25: 9040-9053, 2005.

5. Okamoto I, Kawano Y, Mastsumoto M, Suga M, Kaibuchi K, Ando M and Saya H: Regulated CD44 cleavage under the control of protein kinase C, calcium influx, and the Rho family of small G proteins. J Biol Chem 274: 25525-25534, 1999.

6. Kajita M, Itoh Y, Chiba T, Mori H, Okada A, Kinoh H and Seiki M: Membrane-type 1 matrix metalloproteinase cleaves CD44 and promotes cell migration. J Cell Biol 53: 893-904, 2001.

7. Pelletier L, Guillaumot P, Freche B, et al: Gamma-secretasedependent proteolysis of CD44 promotes neoplastic transformation of rat fibroblastic cells. Cancer Res 66: 3681-3687, 2006.

8. Ito K, Okamoto I, Araki N, et al: Calcium influx triggers the sequential proteolysis of extracellular and cytoplasmic domains of E-cadherin, leading to loss of $\beta$-catenin from cell-cell contacts. Oncogene 18: 7080-7090, 1999.
9. Marambaud P, Shioi J, Serban G, et al: A presenilin-1/gammasecretase cleavage releases the E-cadherin intracellular domain and regulates disassembly of adherens junctions. EMBO J 2: 1948-1956, 2002.

10. Moh MC, Lee LH and Shen S: Cloning and characterization of hepaCAM, a novel Ig-like cell adhesion molecule suppressed in human hepatocellular carcinoma. J Hepatol 42: 833-841, 2005.

11. Moh MC, Zhang T, Lee LH and Shen S: Expression of hepaCAM is downregulated in cancers and induces senescence-like growth arrest via a p53/21-dependent pathway in human breast cancer cell. Carcinogenesis 29: 298-305, 2008.

12. Moh MC, Zhang C, Luo C, Lee LH and Shen S: Structural and functional analyses of a novel Ig-like cell adhesion molecule, hepaCAM, in the human breast carcinoma MCF7 cells. J Biol Chem 280: 27366-27374, 2005.

13. Moh MC, Tian Q, Zhang T, Lee LH and Shen S: The immunoglobulin-like cell adhesion molecule hepaCAM modulates cell adhesion and motility through direct interaction with the actin cytoskeleton. J Cell Physiol 219: 382-391, 2009.

14. Richardson A, Malik RK, Hildebrand JD and Parsons JT: Inhibition of cell spreading by expression of the C-terminal domain of focal adhesion kinase (FAK) is rescued by coexpression of Src or catalytically inactive FAK: a role for paxillin tyrosin phosphorylation. Mol Cell Biol 17: 6906-6914, 1997.

15. Nishizuka Y: The molecular heterogeneity of protein kinase $C$ and its implications for cellular regulation. Nature 334: 661$665,1988$.

16. Bootman MD and Berridge MJ: The elemental principles of calcium signaling. Cell 83: 675-678, 1995.

17. Steinhilb ML, Turner RS and Gaut JR: The protease inhibitor, MG132, blocks maturation of the amyloid precursor protein Swedish mutant preventing cleavage by $\beta$-secretase. J Biol Chem 276: 4476-4484, 2001.

18. Murakami D, Okamoto I and Nagano O: Presenilin-dependent $\gamma$-secretase activity mediates the intramembranous cleavage of CD44. Oncogene 22: 1511-1516, 2003.

19. Wu M, Yu Z, Fan J, Caron A, Whiteway M and Shen SH: Functional dissection of human protease $\mu$-calpain in cell migration using RNAi. FEBS Lett 580: 3246-3256, 2006.

20. Wang KK: Calpain and caspase: can you tell the difference? Trends Neurosci 23: 20-26, 2000.

21. Malen AL, Laurie AS and Priscilla AS: Cathepsin B mediates cleavage of Herpes Simplex virus type 1 origin binding protein (OBP) to yield OBPC-1, and cleavage is dependent upon viral DNA replication. J Virol 81: 9175-982, 2007.

22. Goulet B, Baruch A, Moon NS, et al: A cathepsin L isoform that is devoid of a signal peptide localizes to the nucleus in $S$ phase and processes the CDP/Cux transcription factor. Mol Cell 14: 207-219, 2004.

23. Hooper NM, Karran EH and Turner AJ: Membrane protein secretases. Biochem J 321: 265-279, 1997.

24. Werb Z and Yan Y: A cellular striptease act. Science 282: 1279-1280, 1998.

25. Clemens MJ, Trayner I and Menaya J: The role of protein kinase $\mathrm{C}$ isoenzymes in the regulation of cell proliferation and differentiation. J Cell Sci 103: 881-887, 1992.

26. Rivett AJ: Proteasomes: multicatalytic proteinase complexes. Biochem J 291: 1-10, 1993.

27. Sorokin AV, Selyutina AA and Skabkin MA: Proteasomemediated cleavage of the Y-box-binding protein 1 is linked to DNA-damage stress response. EMBO J 24: 3602-3612, 2005.

28. Baugh JM and Pilipenko EV: 20S proteasome differentially alters translation of different mRNA via the cleavage of eIF4F and eIEF3. Mol Cell 16: 575-586, 2004.

29. De Strooper B, Annaert W, Cupers P, et al: A presenilin-1dependent gamma-secretase-like protease mediates release of Notch intracellular domain. Nature 398: 518-522, 1999.

30. Brown MS, Ye J, Rawson RB and Goldstein JL: Regulated intramembrane proteolysis: a control mechanism conserved from bacteria to humans. Cell 100: 391-398, 2000.

31. Struhl G and Adachi A: Requirements for presenilin-dependent cleavage of Notch and other transmembrane proteins. Mol Cell 6: 625-636, 2000.

32. Xia W and Wolfe MS: Intramembrane proteolysis by presinilin and presenilin-like proteases. J Cell Sci 116: 2839-2844, 2003.

33. Tsubuki S, Saito Y, Tomioka M, Ito H and Kawashima S: Differential inhibition of calpain and proteasome activities by peptidyl aldehydes of di-leucine and tri-leucine. J Biochem 119: 572-576, 1996 . 
34. Pandiella A and Masague J: Multiple signals activate cleavage of the membrane transforming growth factor alpha precursor. J Biol Chem 266: 5769-5773, 1991.

35. Huang EJ, Nocka KH, Buck J and Besmer P: Differential expression and processing of two cell associated forms of the kit-ligand: KL-1 and KL-2. Mol Biol Cell 3: 349-362, 1992.

36. Yee NS, Langen $\mathrm{H}$ and Besmer P: Mechanism of kit ligand, phorbol ester, and calcium-induced down-regulation of c-kit receptors in mast cells. J Biol Chem 268: 14189-14201, 1993.

37. Dethlefsen SM, Raab G, Moses MA, Adam RM, Klagsbrum M, and Freeman MR: Extracellular calcium influx stimulates metalloproteinase cleavage and secretion of heparin-binding EGF-like growth factor independently of protein kinase C. J Cell Biochem 69: 143-153, 1998
38. Mao X, Seidlitz E, Ghosh K, Murakami Y and Ghosh HP: The cytoplasmic domain is critical to the tumor suppressor activity of TSLC1 in non-small cell lung cancer. Cancer Res 63: 7979-7985, 2003.

39. Jiang H, Peterson RS, Wang W, Barinik E, Knudson CB and Knudson W: A requirement for the CD44 cytoplasmic domain for hyaluronan binding, pericellular matrix assembly, and receptor-mediated endocytosis in COS-7 cells. J Biol Chem 277: 10531-10538, 2002. 